\title{
Hanseníase em adultos na região metropolitana de Belém: análise da classificação do grau de incapacidade física e operacional
}

Leprosy in adults in the metropolitan region of Belém: analysis of the classification of the degree of physical and operational disability

Lepra en adultos en la región metropolitana de Belém: análisis de la clasificación del grado de discapacidad física y funcional

Recebido: 20/01/2022 | Revisado: 28/01/2022 | Aceito: 29/01/2022 | Publicado: 31/01/2022

\author{
Tanise Nazaré Maia Costa \\ ORCID: https://orcid.org/0000-0003-1319-5591 \\ Centro Universitário do Estado do Pará, Brasil \\ E-mail: tanise.costa@prof.cesupa.br \\ Cybelle Cristina Pereira \\ ORCID: https://orcid.org/0000-0003-3351-5257 \\ Centro Universitário do Estado do Pará, Brasil \\ E-mail: cybellecpereira@gmail.com \\ Elaine Vilhena de Freitas \\ ORCID: https://orcid.org/0000-0003-3987-6555 \\ Centro Universitário do Estado do Pará, Brasil \\ E-mail: elainefreitasv@gmail.com \\ Guilherme Imbiriba Lisboa Neto \\ ORCID: https://orcid.org/0000-0002-0162-5315 \\ Centro Universitário do Estado do Pará, Brasil \\ E-mail: guilhermelisboa.03@gmail.com e-mail \\ Isabela Martins Viana \\ ORCID: https://orcid.org/0000-0002-3422-856X \\ Centro Universitário do Estado do Pará, Brasil \\ E-mail: isabela.martins.viana@gmail.com \\ Laura Correa de Souza \\ ORCID: https://orcid.org/0000-0002-2315-610X \\ Centro Universitário do Estado do Pará, Brasil \\ E-mail: lauracorrea_souza@hotmail.com \\ Neylane Araújo Cordeiro \\ ORCID: https://orcid.org/0000-0002-4944-6084 \\ Centro Universitário do Estado do Pará, Brasil \\ E-mail: neylane21250215@aluno.cesupa.br \\ Yasmin Batista Mendes \\ ORCID: https://orcid.org/0000-0003-3713-0936 \\ Centro Universitário do Estado do Pará, Brasil \\ E-mail: ybmmendes@gmail.com
}

\begin{abstract}
Resumo
O estudo atual objetivou analisar dados clínicos da classificação do grau de incapacidade física e operacional da doença no estado do Pará, especificamente Região Metropolitana de Belém (RMB) entre adultos de 20-50 anos no período de 2016 a 2020 para obter informações sobre a doença, a fim de entender seu comportamento e fornecer dados atualizados para os profissionais da equipe de saúde. Possui caráter descritivo, indireto, observacional e retrospectivo por intermédio de dados obtidos pelo site do Ministério da Saúde. A hanseníase é um problema de saúde pública no Brasil, destaca-se quase exclusivamente em países em desenvolvimento. Observou-se maior prevalência de diagnósticos de grau de classificação 0 e de classificação operacional multibacilar da doença nos quatro anos estudados e em todos os municípios. mediante esta pesquisa, buscou-se colher informações sobre a infecção em questão, a fim de informar como os números dessa patologia tem impacto na qualidade de vida no Estado do Pará e a importância do seu combate para a melhora dos quadros de saúde da população local.
\end{abstract}

Palavras-chave: Hanseníase; Estratégia Saúde da Família; Doenças transmissíveis.

\begin{abstract}
The current study aimed to analyze clinical data on the classification of the degree of physical and operational disability of the disease in the state of Pará, specifically the Metropolitan Region of Belém (RMB) among adults aged
\end{abstract}


20-50 years in the period from 2016 to 2020 to obtain information about the disease. , in order to understand their behavior and provide updated data to healthcare professionals. It has a descriptive, indirect, observational and retrospective character through data obtained from the Ministry of Health website. Leprosy is a public health problem in Brazil, it stands out almost exclusively in developing countries. There was a higher prevalence of diagnoses of grade 0 classification and multibacillary operational classification of the disease in the four years studied and in all municipalities. Through this research, we sought to collect information about the infection in question, in order to inform how the numbers of this pathology have an impact on the quality of life in the State of Pará and the importance of combating it for the improvement of the health conditions of the local population.

Keywords: Leprosy; Family Health Strategy; Communicable diseases.

\section{Resumen}

El presente estudio tuvo como objetivo analizar datos clínicos de la clasificación del grado de discapacidad física y operativa de la enfermedad en el estado de Pará, específicamente en la Región Metropolitana de Belém (RMB) entre adultos de 20 a 50 años en el período de 2016 a 2020 para obtener información sobre la enfermedad, con el fin de comprender su comportamiento y brindar datos actualizados a los profesionales de la salud. Tiene carácter descriptivo, indirecto, observacional y retrospectivo a través de datos obtenidos del sitio web del Ministerio de Salud.La lepra es un problema de salud pública en Brasil, se destaca casi exclusivamente en los países en desarrollo. Hubo mayor prevalencia de diagnósticos de clasificación grado 0 y clasificación operativa multibacilar de la enfermedad en los cuatro años estudiados y en todos los municipios. A través de esta investigación, buscamos recopilar información sobre la infección en cuestión, con el fin de informar cómo los números de esta patología tienen un impacto en la calidad de vida en el Estado de Pará y la importancia de combatirla para la mejora de la salud. condiciones de la población local.

Palabras clave: Lepra; Estrategia de Salud Familiar; Enfermedades transmisibles.

\section{Introduçãa}

A hanseníase, doença infecciosa crônica causada pelo Mycobacterium leprae, afeta principalmente a pele, os nervos periféricos, as superfícies mucosas do trato respiratório superior e os olhos. É conhecida por ocorrer em todas as idades, desde a primeira infância até a idade avançada. A doença evolui de maneira crônica, podendo apresentar períodos de agudização denominados reações. É potencialmente incapacitante e, embora curável, seu diagnóstico causa grande impacto psicossocial (Azulay, 2013; Cruz et al., 2019).

Essa repercussão psicossocial ocorre, justamente, por ser uma patologia espelhada como um emblema de estigmas, discriminação e preconceito pela sociedade de longa data, a despeito do atual conhecimento e abordagem disseminada acerca do assunto. Adiciona-se ao fato de que o próprio indivíduo doente sofre o afastamento social, vergonha de si mesmo e receio em morrer (Souza \& Martins, 2018).

O período de incubação do bacilo da doença após invadir o organismo é de 2 a 5 anos. O indivíduo infectado, dependendo da resistência natural, pode desenvolver uma das várias formas da doença (Bogliolo, 2011).

De acordo com a Organização Mundial da Saúde (OMS), os doentes são classificados em paucibacilares (PB - com 1 a 5 lesões cutâneas, sem presença demonstrada de bacilos em baciloscopia) ou multibacilares (MB - com mais de cinco lesões de pele; ou com envolvimento dos nervos; ou com a presença demonstrada de bacilos em esfregaço de pele, independentemente do número de lesões cutâneas) (Cruz et al., 2019).

Tal bactéria é transmitida pelas vias respiratórias e estima-se que a maioria da população possua defesa natural contra o M. leprae. Acredita-se que a susceptibilidade ao M. leprae possui influência genética. Assim, familiares de pessoas com hanseníase possuem maior chance de adoecer (Ministério da Saúde, 2017).

Em um contexto histórico, a hanseníase foi identificada no ano de 1873 pelo cientista Armauer Hansen e é uma das doenças mais antigas, com registro de casos há mais de 4000 anos, na China, Egito e Índia (Ferreira, 2019). A doença antigamente era chamada de Lepra e remonta à idéia de impureza e sujeira, contida desde os escritos bíblicos e nas cerimônias de exclusão da Idade Média. A lepra foi no Brasil, durante praticamente todo o século XX, alvo de atenção que a elegeu como uma das grandes 'mazelas' nacionais, segundo os sanitaristas na década de 1910 (Ferreira, 2019). 
No entanto, em 1995, como tentativa de minimizar o estigma de preconceito atribuído à doença, o termo lepra foi substituído por hanseníase oficializado no país, pela Lei nº 9.010, de 29 de março de 1995 (Lopes et al., 2020).

Conforme determinações, o diagnóstico e definição de caso de hanseníase para tratamento apresenta um ou mais dos seguintes achados: lesão de pele com alteração de sensibilidade, espessamento de tronco nervoso e baciloscopia positiva na pele (Faria \& Calábria, 2017; Santos \& Ignotti, 2020).

Outrossim, o tratamento específico da pessoa com hanseníase feito pela atenção básica é a poliquimioterapia (PQT) padronizada pela Organização Mundial de Saúde. A PQT é constituída pelo conjunto dos seguintes medicamentos: rifampicina, dapsona e clofazimina, com administração associada, tendo diferenciação de uma dose mensal supervisionada de rifampicina tomada em 6 meses para os casos PB e de uma dose mensal supervisionada de rifampicina tomada em 12 meses para os casos MB. Além dos medicamentos da PQT, medidas como diagnóstico precoce, prevenção e o tratamento das incapacidades físicas são realizados pelas unidades de saúde atualmente (Ministério da Saúde, 2017).

Vista a partir do quadro nacional tal infecção está estreitamente relacionada às condições econômicas, sociais e ambientais desfavoráveis. A doença é somente considerada endêmica em países onde a prevalência é superior a dez casos por 100 mil habitantes. O Brasil é um desses países (Cruz et al., 2019).

Segundo a OMS o país ocupa o segundo lugar mundial no número de casos de hanseníase, ficando atrás apenas da Índia. Em 2017, no Brasil, foram detectados 26.875 casos novos, o que expressa 12,9 casos a cada 100 mil habitantes (OMS, 2019). No país, o que se observa é que, embora haja empenho de eliminação da hanseníase, as disparidades regionais resultam na manutenção da doença circulante. A grande extensão territorial brasileira e as desigualdades socioeconômicas entre regiões têm sido apontadas como os principais motivos (Ribeiro, Silva \& Oliveira, 2018).

De acordo com o site Agência Brasil o maior número de casos novos identificados na última década está na Região Nordeste, em seguida vêm o Centro-Oeste, Norte, Sudeste e Sul do país. Diante desse cenário, o Brasil é classificado como um país de alto registro endêmico para a doença (Rodrigues et al., 2020).

Em um âmbito regional, segundo o Ministério da Saúde, o Pará foi o quarto em número de pessoas com hanseníase em 2015, apresentando a taxa de detecção geral de novos casos em 35,2/100 mil habitantes (Cunha et al.., 2019; Neves et al., 2017). No município de Belém, como em toda a Região Amazônica, a hanseníase é mais comum em locais com baixa qualidade de vida. Assim, a doença tem apresentado um padrão de endemicidade alto" (Gonçalves et al., 2018). Neste estado, as regiões com maiores riscos são o Sudeste e Centro-Sul, provavelmente, como alguns pesquisadores referem devido à construção da rodovia BR-153 (Rodovia Belém-Brasília), iniciada na década de 1970 e considerada um avanço importante para a expansão da fronteira agrícola no Brasil (Rodrigues et al., 2020).

Com a pandemia do COVID-19 no país, foi perceptível a priorização de atendimento e ações voltadas para os casos do novo coronavírus, e a diminuição da procura e até mesmo de rastreio de casos de sintomáticos dermatológicos de Hanseníase nas unidades de saúde públicas (Silva et al., 2021; Veras er al., 2020).

Considerando que a hanseníase é um problema de saúde pública, o estudo atual objetivou analisar dados clínicos da classificação do grau de incapacidade física e operacional da doença no estado do Pará, especificamente Região Metropolitana de Belém (RMB) entre adultos de 20-50 anos no período de 2016 a 2020, a fim de adquirir um arcabouço teórico sobre a doença, com intuito de levar aos profissionais de saúde propostas de ações de prevenção contra a hanseníase entre os moradores da área.

\section{Metodologia}

O trabalho foi realizado através do levantamento de dados no DATASUS de hanseníase na RMB (compreende municípios de Belém, Ananindeua, Marituba, Benevides e Santa Bárbara), no Estado do Pará, entre os anos de 2016 e 2020. 
Foram incluídas variáveis como: incapacidade física e a classificação operacional dos municípios nos últimos 5 anos.

Não foi necessário o uso do Termo de Consentimento Livre e Esclarecido (TCLE) tampouco submissão ao CEP por se tratar de um estudo descritivo de dados já existentes em bancos de dados públicos. Ademais, não foi imprescindível o Termo de Consentimento de Uso de Dados (TCUD), por se tratar de uma revisão de dados do Ministério da Saúde.

\section{Resultados}

Após levantamento dos dados, em todos os municípios quanto a avaliação do grau de incapacidade física, foi encontrado predominância do Grau 0, conforme a Tabela 1.

Tabela 1: Município de notificação e avaliação de incapacidade física de 2016 a 2020.

\begin{tabular}{|c|c|c|c|c|c|c|}
\hline Município & Branco & GRAU 0 & GRAU 1 & GRAU 2 & Não avaliado & Total \\
\hline Ananindeua & 3 & 192 & 55 & 26 & 26 & 302 \\
\hline Belém & 3 & 656 & 317 & 73 & 131 & 1180 \\
\hline Benevides & 2 & 37 & 21 & 9 & 2 & 71 \\
\hline Marituba & 18 & 1242 & 614 & 318 & 22 & 2214 \\
\hline Santa Bárbara do Pará & 0 & 6 & 5 & 1 & 1 & 13 \\
\hline
\end{tabular}

Fonte: DATASUS.

No que tange a classificação operacional de cada município notificado, a maioria foi da forma multibacilar, conforme demonstra a Tabela 2.

Tabela 2: Município de notificação e classificação operacional atual de 2016 a 2020.

\begin{tabular}{|c|c|c|c|}
\hline Município & PAUCIBACILAR & MULTIBACILAR & Total \\
\hline Ananindeua & 65 & 237 & 302 \\
\hline Belém & 303 & 877 & 1180 \\
\hline Benevides & 10 & 61 & 71 \\
\hline Marituba & 248 & 1966 & 2214 \\
\hline Santa Bárbara do Pará & 3 & 10 & 13 \\
\hline
\end{tabular}

Fonte: DATASUS.

\section{Discussão}

Os casos de hanseníase na RMB podem ser atribuídos à alta densidade demográfica e existência de melhores centros de referências de saúde na área, o que favorece a detecção de novos casos, assim como o manejo e manutenção de todos os pacientes registrados no SINAN.

Esses dados analisados, no período de 2016 a 2020, contaram com foco na atenção das peculiaridades dos municípios que influenciam em relação da expressão da hanseníase, pois ao que se refere a população, em situação econômica e condições de moradia precária, observa-se maior disseminação da doença.

O clima quente e úmido do estado, a localização geográfica na floresta amazônica que é uma região endêmica de algumas doenças e a grande extensão territorial do estado são alguns fatores que influenciam no aparecimento da doença. $\mathrm{O}$ nível sócio-econômico da população relacionado com os baixos dados de escolaridade, são agravantes para o desenvolvimento e o elevado número de casos da doença no estado, haja vista que no Pará há grupos que possuem menor acesso ao tratamento 
por conta de dificuldades geográficas e/ou territoriais, como a população indígena, ribeirinha e quilombola, o que contribui para o número expressivo de casos entre a população paraense.

É importante conhecer não somente essas peculiaridades, mas também entender o comportamento clínico notificado nestes municípios. Em se tratando disso, a portaria conjunta 125/2009 do MS ressalta a questão da classificação da doença quanto aos graus de incapacidade física que deve ser avaliada no momento do diagnóstico de hanseníase e/ou do estado reacional. Para tanto, é necessário realizar o teste da sensibilidade dos olhos, mãos e pés por meio do uso do conjunto de monofilamentos de Semmes-Weinstein (6 monofilamentos) em mãos e pés e do fio dental para os olhos.

Quanto a essa classificação, grau 0 significa que não há nenhuma alteração com os olhos, mãos e pés; grau 1 diz respeito a redução ou perda da sensibilidade nos olhos, mãos e /ou pés; e, grau 2 quando existe lagoftalmo e/ou ectrópio; triquíase; opacidade corneana central; acuidade visual menor que 0,1 ou incapacidade de contar dedos a 6 metros de distância, lesões tróficas e/ou lesões traumáticas em mãos e pés com garras, mão ou pé caído, contratura de tornozelo (Ministério da Saúde, 2009).

O MS considera o coeficiente de detecção de casos novos diagnosticados com grau 2 de incapacidade como um parâmetro importante a ser analisado. De acordo com as tabelas do DATASUS nota-se na maioria dos municípios abrangidos na RMB, destaque aos números da doença em grau 0 e 1 . Essa variação é fundamental, pois, demonstra que as pessoas de grau 1 tem coeficientes maiores de melhora e cura da doença, portanto, de alguma forma o diagnóstico ainda tem sido precoce. A região Norte se destacou como a única que apresentou aumento do coeficiente, de 1,76 em 2005 para 1,83/100 000 habitantes em 2015 (Ribeiro et al., 2018); nesse estudo, ratifica-se quão diferente das outras regiões do país.

A despeito do diagnóstico ocorrendo mais rapidamente, quanto a classificação operacional, encontrou-se um predomínio das formas multibacilares nas cidades da RMB. Essa maior propensão é um indicador de tempo prolongado de adoecimento e de maior possibilidade de transmissão na comunidade denotando menor adesão ao serviço terapêutico disponibilizado pelo SUS pelos pacientes ou até mesmo redução de busca ativa ao tratamento e acompanhamento dos doentes pela equipe de profissionais de saúde das unidades básicas.

Esses dados demonstram que a gestão governamental precisa estar atenta as ações programáticas contra a hanseníase principalmente nos municípios endêmicos com a finalidade de recrutar os trabalhadores da rede básica para auxiliar na eliminação dessa patologia.

O estudo de Garbelini et al., em 2020, no estado de Goiás discorda da presente pesquisa, pois houve uma preponderância de 3,8 vezes de pessoas infectadas com a forma paucibacilar (400\% em relação à multibacilar) e de 4,3 vezes no ano de 2018. Já o trabalho de Santos et al. (2017), no estado do Mato Grosso, assim como de Bucater e Carmo Dias (2020), em São Paulo, encontraram dados semelhantes a presente pesquisa.

Interessante referir que ao analisar isoladamente as notificações por ano, foi observado no último ano de 2020, o número de casos notificados reduziu quase pela metade nos municípios da RMB e, possivelmente, isso se deve à pandemia vivenciada da covid 19, que permite inferir que afetou diretamente na busca ativa e passiva pelo diagnóstico precoce de hanseníase pois muitos usuários das UBS podem ter evitado idas às unidades por questões de isolamento social e até mesmo medidas restritivas de lockdown de contenção ao crescente avanço da pandemia. Além disso, muitos profissionais podem ter se concentrado em diagnosticar precocemente as pessoas infectadas pelo coronavírus, o que pode os ter feito "esquecer", temporariamente, de outras doenças típicas da região paraense.

Destarte, ao decorrer da análise dos casos de hanseníase notificados ao longo desses últimos cinco anos, verificou-se a necessidade de melhorar as atividades de promoção e prevenção à saúde, especialmente, porque essas evidências mostraram maior vulnerabilidade na detecção dos casos, sinalizando uma possível subnotificação da hanseníase por parte da capacitação dos profissionais da saúde. Além disso, há de se considerar que esses fatores influenciam no tempo diagnóstico do paciente, e 
por conseguinte, no seu tratamento porque o quesito tempo diagnóstico é essencial para a evolução do patógeno e, assim, casos da forma clínica da doença.

Esses achados devem inspirar futuros trabalhos semelhantes ou mais pormenorizados para alertar comunidade, governantes e diversos segmentos da sociedade sobre a importância dessa doença por muito tempo considerada "impureza" e causadora de estigmas insuperáveis aos pacientes.

\section{Conclusão}

Conclui-se que essa enfermidade, durante os últimos 5 anos, na RMB, teve maior prevalência de diagnósticos de grau de incapacidade física 0 ou 1 da doença e de classificação operacional multibacilar. Em vista disso, é importante salientar o quão necessário seria uma maior interação dos profissionais da saúde com a comunidade, na medida em que deveriam ocorrer palestras e orientações coletivas, com objetivo de orientar os pacientes sobre a doença, além de elaborar medidas de conscientização sobre a prevenção e tratamento, tendo em vista que esse acometimento merece atenção redobrada devido diversas consequências deletérias.

Sendo assim, mediante esta pesquisa, buscou-se colher informações sobre a infecção em questão, a fim de informar como os números dessa patologia tem impacto na qualidade de vida no Estado do Pará e a importância do seu combate para a melhora dos quadros de saúde da população local. Estudos semelhantes são importantes para alterar a dinâmica ainda existente da patologia.

\section{Referências}

Azulay, R. et al. (2013). Dermatologia, (6a ed.), Ed. Guanabara Koogan. 950 p.

Brasileiro Filho, G. (2011). Bogliolo Patologia, (8a ed.), Ed. Guanabara Koogan. 1288 p.

Bucater, E. P., \& do Carmo Dias, M. A. (2020). Prevalência de casos de hanseníase no município de Votuporanga (SP) no período de 2014 a 2018. Revista Brasileira Multidisciplinar, 23(2), 94-106.

Cruz, G. G. da, Macedo, P. D. O., Dourado, T. L., Silva, I. H. S. da, \& Nunes, R. F. (2019). Estudo Epidemiológico das Formas Clínicas de Hanseníase: Um Panorama Histórico e Atual. Revista Saúde Multidisciplinar, 6(2).

Cunha, D. V., Rodrigues, E. B., Lameira, H. A., da Cruz, M. T. S., Rodrigues, S. M., \& dos Santos, F. D. S. (2019). Perfil Epidemiológico da Hanseníase no Município de Castanhal-Pará no período de 2014 a 2017. Revista Eletrônica Acervo Saúde, 11(15), e858-e858.

Faria, L. \& Calábria, L. K. (2017). Aspectos históricos e epidemiológicos da hanseníase em Minas Gerais. Revista de Medicina e Saúde de Brasília, 6(3). Ferreira, I. N. (2019). Um breve histórico da hanseníase. Humanidades e Tecnologia (FINOM), 16(1), 436-454.

Garbelini, G. U., Paiva, I. G., Appollonio Filho, P., Ferreira, L. P. M., de Oliveira, A. V., Santiago, J. J. C., \& Cruvinel, F. M. (2020). Análise do perfil epidemiológico das formas de apresentação da hanseníase no estado de Goiás. Brazilian Journal of Health Review, 3(2), $2525-2530$.

Gonçalves, N. V., Alcântara, R. C. C., Júnior, A. D. S. S., Pereira, A. L. R. R., Miranda, C. D. S. C., de Sousa Oliveira, J. S., \& Palácios, V. R. D. C. M. (2018). A hanseníase em um distrito administrativo de Belém, estado do Pará, Brasil: relações entre território, socioeconomia e política pública em saúde, 2007-2013. Revista Pan-Amazônica de Saúde, 9(2), 10-10.

Lopes, E. F. B., da Silva, L. S. A., de Sousa Rotta, C., De Oliveira, J. H. M., de Menezes, I. R., Nakamura, L., \& Simões, E. A. P. (2020). Educação em saúde: uma troca de saberes no combate ao estigma da hanseníase. Brazilian Journal of Development, 6(2), 5350-5368.

Ministério da Saúde - Guia prático sobre a Hanseníase, https://portalarquivos2.saude.gov.br/images/pdf/2017/novembro/22/Guia-Pratico-deHanseniaseWEB.pdf.

Ministério da saúde. (2009). Portaria conjunta nº 125, de 26 de março de 2009. https://bvsms.saude.gov.br/bvs/saudelegis/svs/2009/poc0125_26_03_2009.html

Neves, D. C. D. O., Ribeiro, C. D. T., Santos, L. E. S., \& Lobato, D. D. C. (2017). Tendência das taxas de detecção de hanseníase em jovens de 10 a 19 anos de idade nas Regiões de Integração do estado do Pará, Brasil, no período de 2005 a 2014. Revista Pan-Amazônica de Saúde, 8(1), 29-37.

Organização Mundial da Saúde - Diretrizes para o diagnóstico, tratamento e prevenção da hanseníase 2019. https://apps.who.int/iris/bitstream/handle/10665/274127/9789290227076por.pdf?sequence=47\&isAllowed=y\#: :text=Tratamento\%20da\%20hansen\%C3\%AD ase, $12 \% 20$ meses\%20para\%20hansen\%C3\%ADase\%20MB.

Ribeiro, M. D. A., Silva, J. C. A., \& Oliveira, S. B. (2018). Estudo epidemiológico da hanseníase no Brasil: reflexão sobre as metas de eliminação. Revista Panamericana de Salud Pública, 42, e42. 
Research, Society and Development, v. 11, n. 2, e43911225976, 2022

(CC BY 4.0) | ISSN 2525-3409 | DOI: http://dx.doi.org/10.33448/rsd-v10i2.25976

Rodrigues, R. N., Leano, H. A. D. M., Bueno, I. D. C., Araújo, K. M. D. F. A., \& Lana, F. C. F. (2020). Áreas de alto risco de hanseníase no Brasil, período 2001-2015. Revista Brasileira de Enfermagem, 73.

Santos, A. R. D., \& Ignotti, E. (2020). Prevenção de incapacidade física por hanseníase no Brasil: análise histórica. Ciência \& Saúde Coletiva, 25, 3731-3744.

Santos, D. A. da S., Spessatto, L. B., Melo, L. S., de Olinda, R. A., Lisboa, H. C. F., \& da Silva, M. S. (2017). Prevalência de casos de hanseníase. Revista de Enfermagem UFPE on line, 11(10), 4045-4055.

Silva, J. M. dos S., Nascimento, D. C. do, Moura, J. C. V., Almeida, V. R. S. de, Freitas, M. Y. G. S., Santos, S. D. dos, \& da Silva, I. R. S. (2021). Atenção às pessoas com hanseníase frente a pandemia da covid-19: uma revisão narrativa. Revista Eletrônica Acervo Saúde, 13(2), e6124-e6124.

Souza, A. O. de, \& Martins, M. D. G. T. (2018). Aspectos afetivos e comportamentais do portador de hanseníase frente ao estigma e preconceito. Revista de Iniciação Científica da Universidade Vale do Rio Verde, 8(1). 